\title{
Halogen Lamp Modeling For Low Voltage Power Systems Transient Analyses
}

\author{
O. Craciun, D. Radu, Member, IEEE, S. Bacha, Member, IEEE
}

\begin{abstract}
This paper deals with halogen luminaries, ones of the main used lighting sources in residential area. The use of these artificial light sources leads towards different operational constraints in the low voltage power systems, such as inrush currents, harmonics or high frequency perturbations. Hence, the aim of the work is the characterization and modeling of halogen luminaries in ATP-EMTP, in order to understand low voltage power systems transient phenomena and the conditions of lamps compatibility with the electrical devices such as circuit breakers or residual current protection. Measurements on real lighting power systems were performed to show the accuracy of developed models.
\end{abstract}

Index Terms-halogen lamp, EMTP-ATP, transient analyses, low voltage power systems.

\section{INTRODUCTION}

$\mathrm{N}$ OWADAYS, lighting is essential all across the modern society. It is very hard to find an activity that takes place without it. Lighting is responsible for 14 percents of the European Union's electricity consumption and for up to 60 percents for the commercial buildings. In the same time, improving energy efficiency is crucial for the climate change problem. European Union's goal is to reduce greenhouse gas emission by 20 percents by 2020 [1], [2].

As the lighting sources represent a consequent electrical load, a solution to decrease the electricity consumption in these systems is to replace the lamps with lower efficiency (e.g.: incandescent lamp) by lamps with a higher efficiency such as halogen lamps, compact fluorescent lamps or even LEDs (Light Emitting Diodes). To drive these newer lamps, the use of non-linear devices such as electronic transformers (ETs), toroidal transformers (TTs) or ballasts is necessary. New perturbations are thus introduces in the lighting systems by these devices, such as: inrush currents, harmonics or highfrequency perturbations. Even in the presence of these new problems, the proper exploitation of lighting power systems is required.

Given the circumstances, the interaction between low voltage (LV) power systems and the light sources, such as

O. Craciun and S. Bacha are with G2Elab (Grenoble Electrical Engineering Laboratry), ENSE3, BP 46, 38402 Saint Martin d'Hères Cedex, France (e-mail: octavian.craciun@g2elab.grenoble-inp.fr and seddik.bacha@g2elab.grenoble-inp.fr).

D. Radu is with Schneider Electric Industries (Business Unit Power Systems and Mechatronic Integration), 31 Pierre Mendes St., F-38050, Eybens, France (e-mail: daniel2.radu@fr.schneider-electric.com). halogen luminaries, is necessary to be studied. In the halogen lamp family, two subcategories can be identified: halogen lamps driven directly in LV power systems - low voltage halogen lamps (HLs); extra low voltage halogen lamps (ELHs) driven by a LV/extra low voltage (ELV) conversion device such as TT or ET.

In literature, ATP-EMTP models of TTs [3], [4], [5], [6] for different application such as current transformer were developed. Studies regarding the high-frequency effect on the ELH and the possibility of electromagnetic noise reduction in the case of ET were achieved [7], [8]. In the same time, standard incandescent lamp models and HLs models [9] were developed in ATP-EMTP in order to study the interaction between these loads and the LV power systems. But studies regarding the power up problem of electrical lighting systems where ETs or TTs are associated with halogen light sources were not accomplished.

In this context, this paper investigates the ELV halogen luminaries. The aim of this work is to analyze the transient phenomena that occur in lighting power systems, by modeling and characterization of the HLs, ELHs, TTs and ETs.

\section{HALOGEN LUMINAIRES}

\section{A. Halogen lamp}

The main constitutive element of halogen lamps is the tungsten filament which will produce light by passing an electrical current through it. Halogen lamps can be fed up with LV or ELV power supply. In the case of ELV systems, a LV/ELV conversion device (as TT or ET) is necessary (see Fig. 1).

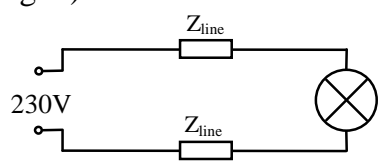

(a)

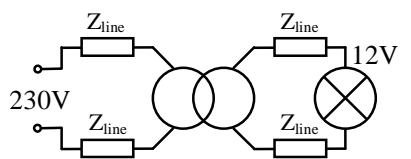

(b)
Fig. 1. Standard halogen lamps electrical circuits: HL (a) and ELH (b).

Halogen lamps are similar to incandescent lamps - the tungsten filament is sealed into a glass bulb filled with an inert gas (e.g.: argon or neon) mixed with a small amount of halogen (e.g.: iodine or bromine) [10]. The halogen reacts with the tungsten vapor in order to form a tungsten halide that circulates in the filament area. The halogen allows the filament to be run at a higher temperature and also allows to deposit tungsten from the inside of the bulb back onto the filament, this increasing the lifetime of the lamp which is estimated to 
up to 4000 hours (the double of the incandescent lamp lifetime) [11], [12]. Fig. 2 shows an example of halogen lamps.

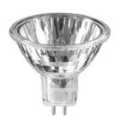

(a)

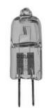

(b)
Fig. 2. Halogen lamps example: reflector spotlight (a) and pinbase lamp (b).

The main advantages of these lamps are instant ignition, low investment cost and frequent switching possibility. Among their disadvantages we note the high electricity consumption, the operation cost and the dimensions of the transformers in the case of ELH. Halogen luminaries are mainly used in homes, restaurants, shops or projectors.

\section{B. Toroidal transformer}

As mentioned, for ELH drive, the use of a LV/ELV conversion device is required. One of the devices used in order to assure this conversion is the TT. These devices transfer electrical energy from the primary winding to the secondary one, without changing the frequency, by using the principle of electromagnetic induction. Usually, the energy transfer takes place with a voltage change. For the considered TT in this study, the $230 \mathrm{~V}$ input voltage is transformed into $12 \mathrm{~V}$ at $50 \mathrm{~Hz}$ (common for the European power). The main constitutive elements of the transformer are represented by the primary and the secondary windings (copper wires) wrapped around a cylindrical core. Fig. 3 shows the cylindrical iron core of a TT.

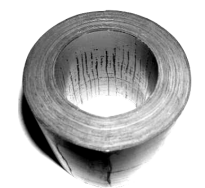

Fig. 3. TT cylindrical iron core.

The main advantages of this device are its small size, low weight, low price and low no-load losses. Also, TTs are easy adaptable to customer requirements.

\section{Electronic transformer}

ET represents an alternative solution of power conversion to the standard iron core, $50 \mathrm{~Hz}$ transformer technique. The advantages of the ET compared with the classical solution are the fact that they have about $60 \%$ of the volume, $15 \%$ of the weight of conventional transformers [13]; the output power from the ET can be varied, and thus dimming control can be added. Another advantage of the ET is that a protection against short circuit of the lamp can be included.

An overview of the general diagram of an ET is given in Fig. 4. Its structure is similar to a classic half-bridge circuit. The input voltage is rectified by the full-bridge rectifier resulting in a semi-sinusoidal voltage at the double of the line frequency. Then, by passing through the inverter, this voltage is converted into a high-frequency one (usually at $35 \mathrm{kHz}$ ) and finally converted back into $12 \mathrm{~V}$. The input filter avoids the radio interferences and also protects the unit from perturbations.

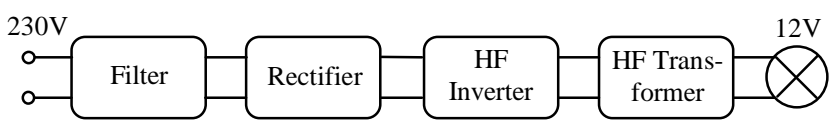

Fig. 4. Principle diagram of an ET which powers a ELH.

Nowadays, the ET represents a more compact and efficient alternative to TT. Its inconvenient remains the price which is up to 5 superior to the classic transformer [14].

\section{HALOGEN LUMINARIES MODELING AND IMPLEMENTATION IN ATP-EMTP}

This section deals with halogen luminaries characterization, modeling and implementation in ATP-EMTP in order to study the problems related to the powering up of a lighting power system where halogen luminaries are associated.

\section{A. Halogen lamp model}

Halogen lamps are producing light by passing an electrical current through the tungsten filament which will heat from an initial value to a final one. As the main constitutive element of a halogen lamp is the tungsten filament, the LV electrical network conceives a halogen lamp as a variable resistance. So, a halogen lamp represents a pure resistive load. According to [9], the physical behavior of the tungsten filament can by represented by the following mathematical model:

$$
R_{\text {lamp }}=R_{i}+\left\lfloor\left(R_{f}-R_{i}\right) \cdot\left(1-e^{-t / \tau}\right)\right\rfloor
$$

where $R_{i}$ represents the initial resistance value, $R_{f}$ represents the final resistance value and $\tau$, the time constant.

In order to identify the model parameters, laboratory tests were performed, as shown in [9]. Fig. 5 shows the resistance variation of a $100 \mathrm{~W}$ HL.

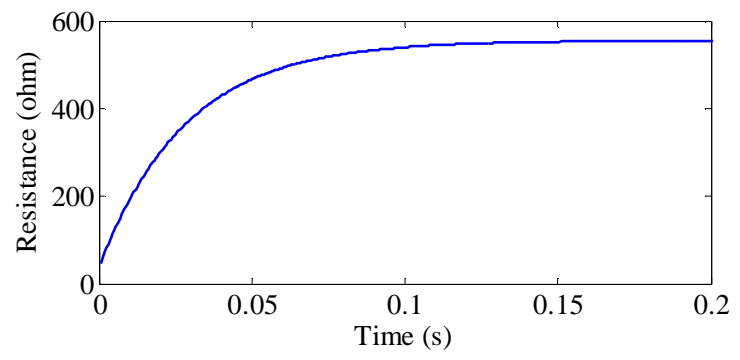

Fig. 5. $100 \mathrm{~W}$ HL resistance variation.

As described in [9], in order to characterize different rated powers of halogen lamps, the model parameter determination was performed for several lamps.

The initial halogen lamp resistance value can be up to $1 / 15$ the steady-state resistance value, resulting in a high inrush current for the first few cycles after switching on. So, the ratio between the inrush current and the steady-state current can be up to 15 . This value mainly depends on the rated power of the lamp and the supply and electrical circuit impedances.

As a halogen lamp mathematical model is developed, its representation in ATP-EMTP is now considered. The model of a HL/ELH in ATP-EMTP will be represented by a Type 91 resistance [15] controlled by a model implemented in ATPModels that represents the mathematical model of the lamp 
variable resistance [9]. A representative icon was chosen in ATP-EMTP for the HL/ELH representation (see Fig. 6).

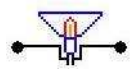

Fig. 6. HL model representation in ATP-EMTP.

\section{B. Toroidal transformer model}

Transformer models are especially important for studying the saturation current, ferroresonance phenomena, harmonics and their effect on the electrical power systems. The major nonlinear effects in iron cores are saturation, eddy currents, and hysteresis. An appropriate TT model based on its saturation characteristic is presented below.

Generally, a single phase two winding transformer can be represented as in the following diagram (see Fig. 7):

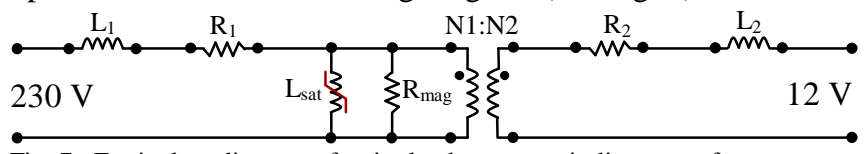

Fig. 7. Equivalent diagram of a single phase two windings transformer.

Both windings have an associated leakage-impedance, characterized by the resistances $R_{1}$ and $R_{2}$ and by the inductances $\mathrm{L}_{1}$ and $\mathrm{L}_{2}$.

The saturation effect is confined to a single nonlinear reactor $\left(\mathrm{L}_{\mathrm{sat}}\right)$. In the ATP-EMTP program, two classes of non-linear inductances are available for the saturation effect representation: a true nonlinear model (Type 93), and two pseudo-nonlinear models (Type 96 and Type 98) [15]. In the Type 93 model, the nonlinearity of the model is represented by a nonlinear function (flux as function of current). In the pseudo-nonlinear model, the nonlinearity is represented by a number of piecewise linear segments. ATP-EMTP represents then these segments as a resistor in parallel with an appropriate current source.

Excitation losses are confined to a linear magnetization resistance, $R_{\text {mag }}$ (see Fig. 7), which is in parallel with the saturation branch.

The considered transformer to be modeled is a 50VA single phase two winding LV/ELV transformer used for the ELH. Its model is developed according to the equivalent single phase transformer electrical diagram (see Fig. 1). The saturation effect will be represented by a Type 96 nonlinear inductance.

For the models parameter identification, open-circuit test and short-circuit test were performed. The TT model parameters are presented in table I.

TABLE I

TT MODEL PARAMETERS

\begin{tabular}{|c|c|c|}
\hline \multicolumn{2}{|c|}{ Data } & Value \\
\hline $\mathrm{R}_{1}$ & {$[\Omega]$} & 52.37 \\
\hline $\mathrm{R}_{2}$ & {$[\Omega]$} & 0.22 \\
\hline $\mathrm{L}_{1}$ & {$[\mathrm{mH}]$} & 14 \\
\hline $\mathrm{L}_{2}$ & {$[\mathrm{mH}]$} & 0.06 \\
\hline $\mathrm{R}_{\mathrm{mag}}$ & {$[\Omega]$} & 13225 \\
\hline
\end{tabular}

For the Type 96 nonlinear inductance parameters determination, the HYSTERESIS subroutine of ATP-EMTP [15] is used. The purpose of this subroutine is to determine the necessary (Flux, Current) characteristic. The input data for this subroutine are current and flux at positive saturation point. As this data are available only from the iron core hysteresis characteristic, SATURATION subroutine [15] is used to determine it, starting from the saturation characteristic.

For the saturation test, the primary winding of the transformer is excitated and voltages and corresponding currents are measured in order to create the (V, I) characteristic (see Fig. 8).

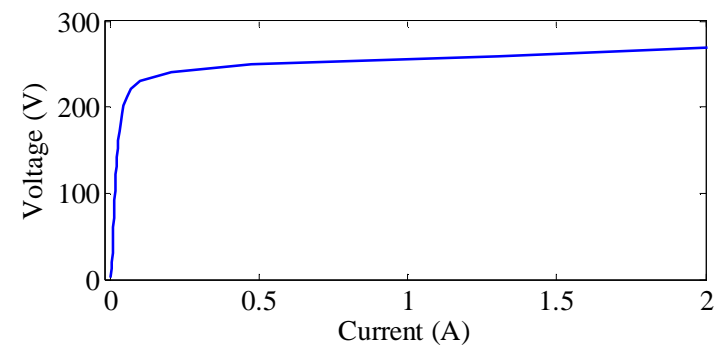

Fig. 8. TT saturation characteristic.

By using the SATURATION subroutine in ATP-EMTP, the (V, I) characteristic is converted into (Flux, Current) characteristic. Fig. 9 shows the implemented SATURATION subroutine and in Fig. 10 presents the resulted peak current as a function of flux characteristic.

BEGIN NEW DATA CASE

\$ERASE

SATURATION

345678901234567890123456789012345678901234567890123456789012

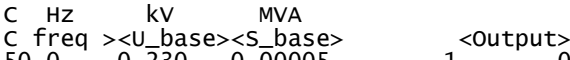

$\begin{array}{llll}50.0 & 0.230 & 0.00005 & 1\end{array}$

C I Irms > U r rms

$0.0013790 \quad 0.00621$

$0.004967 \quad 0.21913$

$\begin{array}{ll}0.004967 & 0.21913 \\ 0.006302 & 0.32739\end{array}$

$0.007773 \quad 0.43826$

$0.009429 \quad 0.54217$

$0.012144 \quad 0.65609$

$0.016605-0.76435$

$\begin{array}{ll}0.026265 & 0.87261 \\ 0.050231 & 0.95696\end{array}$

$0.089236 \quad 1.00000$

1.13260

1.222600

\$PUNCH

9999

1.19090

BLANK LINE ending saturation data

BEGIN NEW DATA CASE

BLANK LINE ENDING ALL CASES

Fig. 9. SATURATION implementation in ATP-EMTP.

$\begin{array}{lll}\text { Row } & \text { Current [amp] } & \text { Flux [volt-sec] } \\ 1 & 0.0000000000 & 0.0000000000 \\ 2 & 0.0004239566 & 0.0064296090 \\ 3 & 0.0009284558 & 0.1137140022 \\ 4 & 0.0013813741 & 0.2268792615 \\ 5 & 0.0017688956 & 0.3389677426 \\ 6 & 0.0022564142 & 0.4537585230 \\ 7 & 0.0028461231 & 0.5613431717 \\ 8 & 0.0039540978 & 0.6792918116 \\ 9 & 0.0059196261 & 0.7913802927 \\ 10 & 0.0105295131 & 0.9034687737 \\ 11 & 0.0236642878 & 0.9908017072 \\ 12 & 0.0489670686 & 1.0353637636 \\ 13 & 0.4721951220 & 1.1726529986 \\ 14 & 0.5505177450 & 1.2330147060\end{array}$

Fig. 10. SATURATION result (peak current as a function of flux).

As the current and the flux at positive saturation point are now available, HYSTERESIS subroutine is then used to provide the necessary hysteresis loop data for the Type-96 pseudo-nonlinear reactor model (see Fig. 11). The resulted (Flux, Current) characteristic is given in Fig. 12. 
BEGIN NEW DATA CASE

HYSTERESIS

\$ERASE

$C$ ITYPE LEVEL

$\begin{array}{rr}1 & 4 \\ 0.489 & 1.035\end{array}$

\$PUNCH

BLANK

BEGIN NEW DATA CASE

BEGIN $N$

Fig. 11. HYSTERESIS implementation in ATP-EMTP.

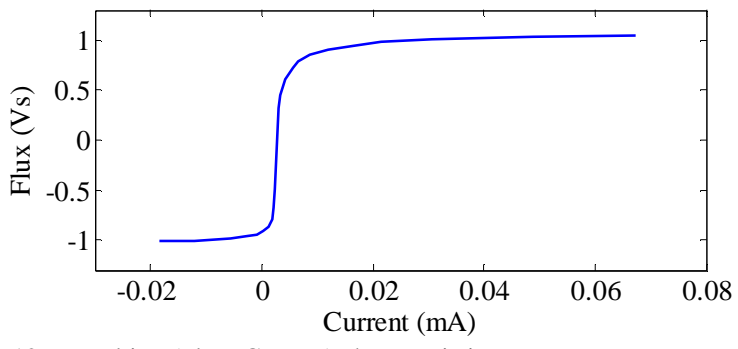

Fig. 12. Resulting (Flux, Current) characteristic.

The (Flux, Current) determined characteristic must be completed with one more point that represents the air-core inductance $L_{\text {air }}$ (the additional flux which circulates in air).

The air-core inductance can be approximated by the geometry of the corresponding winding:

$$
L_{\text {air }}=\mu_{0} \cdot N^{2} \cdot A / l[\mathrm{H}]
$$

where $\mu_{0}$ represents the permeability of free space, $N$ represents the number of turns, $A$ is the cross sectional area of the winding and $l$, the winding length leg length. The air core inductance calculation may be different according to the geometry of the iron core.

The corresponding current value is calculated according to [15] as follows: firstly a $\Delta I$ value is estimated as the ratio between the flux which circulates in air and $L_{\text {air }}$. The air flux is estimated as the difference between the flux in the considered winding at its first maximum and the saturation flux. Finnaly, the peak current value, corresponding to the flux which circulates in the air, is now determined by the addition of $\Delta I$ to the peak current value corresponding to the saturation flux.

As all the necessary parameters for the single phase TT are available, its model can be implemented in ATP-EMTP by following the electrical diagram showed in Fig. 7. The saturation effect is represented by a Type 96 pseudo-nonlinear inductance. As for the HL model, an icon was chosen to represent a TT which powers an ELH (see Fig. 13).

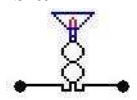

Fig. 13. ATP-EMTP representation of the TT model together with the ELH model.

\section{Electronic transformer model}

As seen in Fig. 4, the main constitutive elements of an ET used for powering up ELHs are the line filter, the rectifier, the high frequency inverter and the high frequency transformer.

The considered ET for this study is a $150 \mathrm{~W}$ one which has a secondary voltage of $11.8 \mathrm{~V}$. The main frequency is $50 \mathrm{~Hz}$ and the operating frequency at around $35 \mathrm{kHz}$.
Before creating the ET ATP-EMTP model, the real transformer was studied in order to identify its constitutive elements and theirs characteristics. Also, by considering the existent classic electrical diagrams of ETs [13], [16], Fig. 14 shows the electrical diagram of an ET.

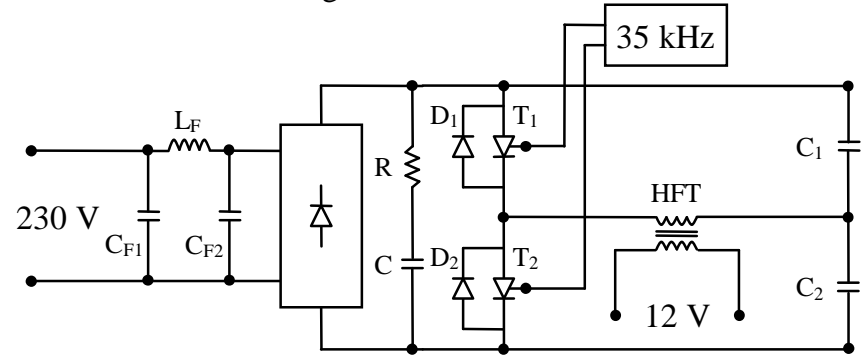

Fig. 14. ET electrical diagram.

The main line voltage is firstly rectified by the full-bridge rectifier and the DC bus capacitor is fully charged. The power stage is a half-bridge inverter, supplied from the power line, which delivers the power to the lamp by passing through the high frequency transformer (HFT). The two inverters transistors are considered in the ATP-EMTP model as a diode in series with a time controlled switch. The transistors are driven in the model, by a pulse train signal source at $35 \mathrm{kHz}$ delayed by an output pulse delay Type 98 [15]. In this kind of applications, the oscillation frequency is around $35 \mathrm{kHz}$, generally around ten times the natural frequency of the circuit. The voltage across the DC bus capacitor permits the transistor to switch. The anti-parallel diodes allow current to flow when the devices are off. Also, in the model, for the diode protection, a snubber circuit was connected in parallel with each of them.

A Type 18 ideal transformer [15] was chosen to represent the high frequency transformer (HFT). This simplifying hypothesis was taken into account because the HFT does not influence the inrush current due to the powering up of a system constituted of an ET and an EHL.

The association of the ET model and the ELH model is represented in ATP-EMTP by the icon presented in Fig. 15.

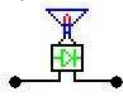

Fig. 15. ATP-EMTP representation of the ET model together with an ELH model.

\section{LABORATORY TESTS AND MODELS VALIDATION}

For the models validation, tests were performed within a test laboratory on a real LV power system where different halogen luminaries are connected. Fig. 16 gives an overview on the test bench main constitutive parts.

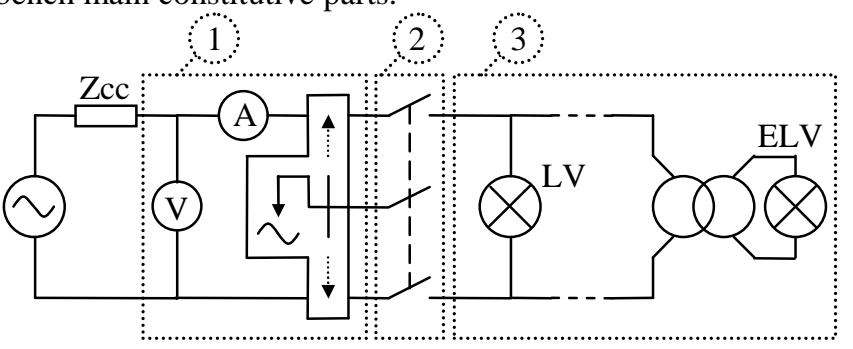

Fig. 16. The test bench for halogen luminaries. 
The test bench consists of three main parts: the first one is represented by the measure equipment and by the device that permits to switch on the circuit at the desired time. Hence, voltage zero crossing instant can drastically change the ratio between the inrush current and the steady state current. This high current peak of inrush current can have adverse effects on the lighting system. It can affect the relay contacts, the circuit breaker, and other related components. Repeated exposure to the stress of inrush current can shorten the operating life span of these elements. For the models validation tests, the zero voltage switching will be considered.

The test bench second unit is constituted of switching equipments (in this case, a contactor and the protection device). The third part is the halogen luminaries unit that permits the connection into the LV power system of several lamps in parallel. This unit allows performing tests on the most used incandescent and HLs and even on compact fluorescent lamps.

To validate the developed models for different case studies, a part of the test laboratory equipments must be modeled and represented in ATP-EMTP. The main constitutive elements of this model are: the power supply together with its short-circuit impedance, the line cables, the switching equipments that are considered as ideal time controlled switches and the LV load represented by a HL and by two ELH powered up by a TT, and secondly by a ET (see Fig. 17). Behind the icons that represent the halogen loads, we find the complete models, described in Section IV.

For the power supply model, an alternative electrical current source Type 14 [15] was considered. Its parameters correspond to a $230 \mathrm{~V}-50 \mathrm{~Hz} \mathrm{LV}$ power system. In series with the power supply, the short-circuit impedance is modeled as an RL branch (the RL values were measures in the test laboratory). Finally, in order to represent the electrical cables in ATP, the PI line model [15] was used.

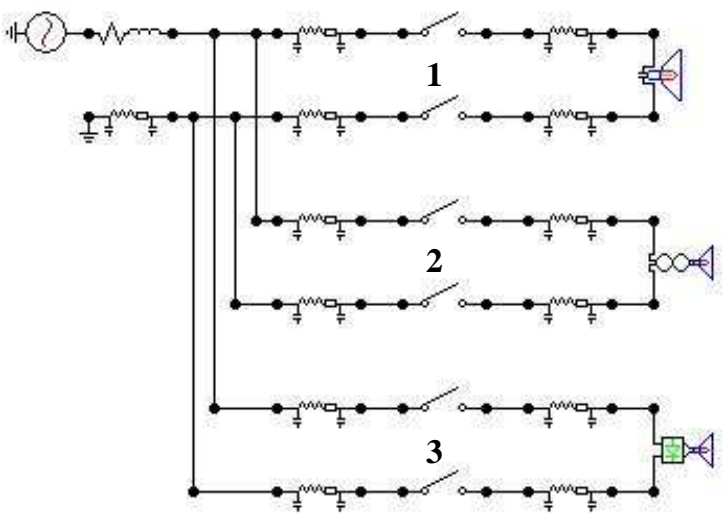

Fig. 17. ATP-EMTP model of the considered electrical network.

For the first test, switch 1 was closed in order to study the powering up of a $100 \mathrm{~W} \mathrm{HL}$. The current in the feeding point to the electrical network was measured and compared to the current evolution obtained in the ATP-EMTP simulation, as shown in Fig. 18.

As expected, the inrush current due to the powering up of a $\mathrm{HL}$ is, in this case, approximately five times bigger then the steady state current. According to the lamps rated power and electrical network characteristics, the ratio between the inrush current and the steady state current can be up to 15. Also, as the electrical current obtained in the simulation is strictly the same with the measured current, we can say that the developed HL model is validated and it permits an exact representation of the transient phenomena that take place into lighting power system.

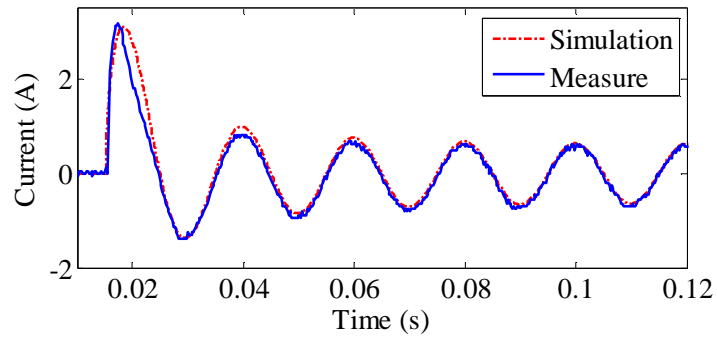

Fig. 18. Inrush current of a 100W HL.

The second test was performed using the same feeding scheme, on the TT. In this case, switch 2 was closed in order to study the inrush current due to the iron core magnetization of the TT. In the secondary winding of the TT, a 50W ELH is connected. The current in the feeding point of the electrical network was measured and compared with the current obtained in the simulation (see Fig. 19).

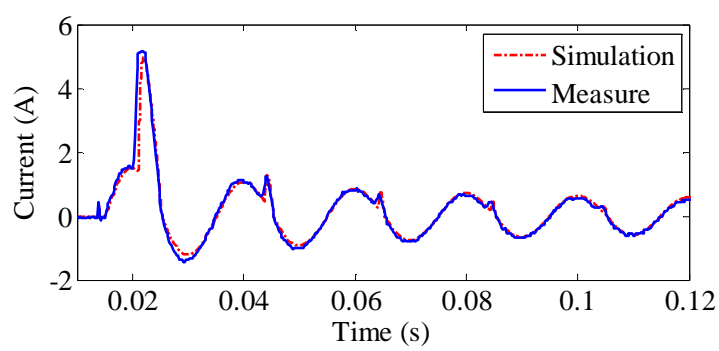

Fig. 19. Inrush current of a $50 \mathrm{~W}$ luminaire (TT\&ELH).

The inrush current due to the powering up of this halogen load can be considered as formed by two inrush currents: the first one represents the transformer magnetization current, and the second one is the current due to the heating of the lamp filament.

In the lighting power systems where TTs are connected, the inrush current can represent to up to 100 times the steady state current (in the case of the connection in the secondary of the transformer an ELH of low rated power, e.g. 5W).

The developed TT model permits an accurate representation of the iron core magnetization and of the problems dues to powering up and to functionalities of lighting power systems.

The last validation test presented in this paper concerns the ET. For that, an ET which supplies a 50W ELH is connected into the considered electrical network by closing switch 3 .

As for the two study cases presented before, the simulated inrush current follows the evolution of the measured one (see Fig. 20). In this case, the inrush current is due to the charge of the circuit and to the lamp resistance variation. The inrush current value can also be up to 100 times bigger then the steady state current value, according to three factors: the switch off moment of the load, the lamp rated power and the electrical network configuration. 


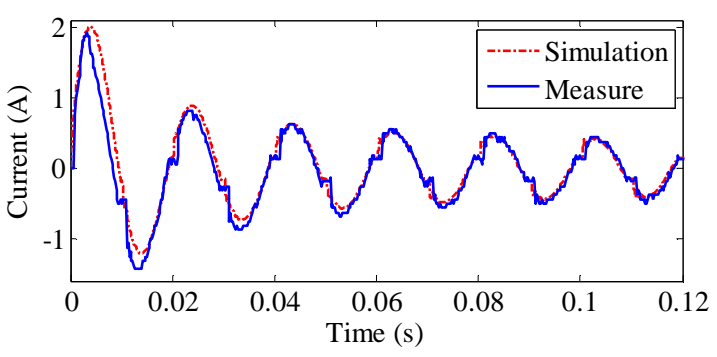

Fig. 20. Inrush current of a $50 \mathrm{~W}$ luminaire (ET\&ELH).

A second verification of this model was performed. The DC bus voltage was measured in order to be compared with the ATP-EMTP simulation (see Fig. 21). The waveform of this voltage is a signal that has the double of the line frequency.

This model can easily represent the transient phenomena that took place in lighting power systems where ETs are associated.

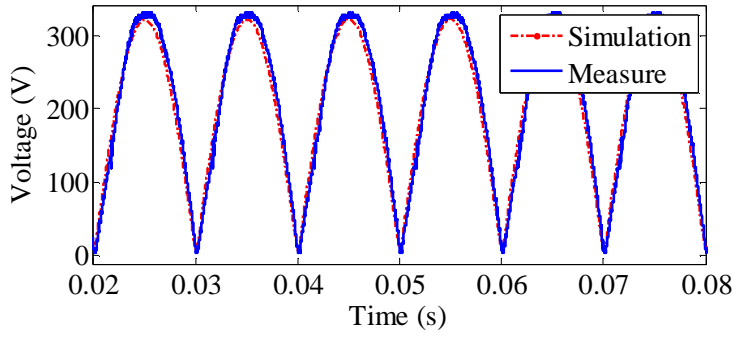

Fig. 21. ET voltage at the DC bus.

Different studies cases were also considered (e.g.: test with several lamps in parallel) in order to validate the developed models. The results obtained are very satisfactory. Only the representative cases were considered to be presented in this article. Also, ATP-EMTP software offers a good environment for the representation of transient phenomena and of different perturbations that take place in the lighting electrical systems, especially in the moment of powering up this kind of loads.

\section{CONCLUSION}

Lighting includes both artificial light (electrical lamps) and natural sources such as natural illumination from daylight. Lighting represents a major component of energy consumption, accounting for a significant part of all energy consumed worldwide. Artificial lighting is most commonly provided today by electric lights.

To study the interaction between the LV power systems and the most used electric light sources, the paper describes models implemented in ATP-EMTP for halogen light sources powered in LV and ELV power systems. The proposed models take into account the physical behavior of the lamps and also the different problems regarding the conversion devices.

Thank to this approach, a precise representation of the problems due to the powering up of these loads was achieved. The aim of this study was to bring new elements for the switching equipment conception and development.

\section{REFERENCES}

[1] The Need Project, "Energy Consumption", Secondary Energy Infobook, Manassas, 2008, pp. 66-71.

[2] European Lamp Companies Federation, "The ELC Roadmap for Deploying Energy Efficient Lighting Technology Across Europe", Brussels, Belgium, 2008, pp 3.

[3] J. A. A. Camarena, "Modeling and Simulation of Current Transformers with the EMTP-ATP" - in Spanish, Latin America Transactions, IEEE (Revista IEEE America Latina), 2004, pp. 201-205.

[4] A. Mechraoui, A. Draou , A. Akkouche, and S. AL Ahmadi, "Current Transformer Performance study Using Software Tools", Saudi Technical Conference, Electrical Power Systems and Machines Technologies, Saudi Arabia, 2006.

[5] Working Group C-5 of the Systems Protection Subcommittee of the IEEE Power System Relaying Committee, "Mathematical Models for Current, Voltage, and Coupling Capacitor Voltage Transformers", IEEE Transactions on Power Delivery, Vol. 15, No. 1, 2000, pp. 62-72.

[6] J. Frame, N. Mohan, T.-H. Liu, "Hysteresis Modeling in An Electromagnetic Transient Program", IEEE Transactions on Power Apparatus and Systems, Vol. PAS-101, No. 9, September 1982, pp. 3403-3412.

[7] C. Blanco, P. Villegas, E. Lopez, J. M. Alonso, M Rico, “An Improved Electronic Transformer for Low-Voltage Halogen Cycle Lamps", IEEE Transactions on Industry Applications, Vol. 3, 1994, pp. 1979-1983.

[8] K. Jirasereeamornkul, I. Boonyaroonate, K. Chamnongthai, "High Efficiency Electronic Transformer for Low Voltage Halogen Lamp", Circuits and Systems, ISCAS, Proceedings of the 2003 International Symposium, 25-28 May 2003, pp. 355-358.

[9] O. Craciun, D. Radu, S. Bacha, "Incandescent Lamp Characterization and Modeling" European ATP-EMTP Conference, Izmir, Turkey, 2008, pp. 188-201.

[10] French Lighting Association, "Light Sources" - in French, LUX Editions Society, 2007, ch. 4.

[11] Schneider Electric Industries, "Lighting Circuits - Selection, Dimensioning and Practical Recommendations", Rueil-Malmaison, 2008, pp 6-7.

[12] R. S. Simpson, "Lighting Control - Technology and Applications", Focall Pres, 2003, ch. 3.

[13] P. Fichera, R. Scollo, "Electronic Transformer for a 12V Halogen Lamp", AN528/0999, ST Microelectronics Group of Companies.

[14] Osram, "Price billing 2008 - Devices, Lamps, Luminaries for the General Lightning" - in French, Molsheim, France, 2008.

[15] W. Scott Meyer, Tsu-Huei Liu, "ATP Rule Book”, Canadian/American EMTP User Group, 1987.

[16] P. Green, "Electronic Transformer Applications", Application Note AN1069, International Rectifier, California, June 2005.

\section{BIOGRAPHIES}

Octavian Craciun was born in 1983 in Bucharest, Romania. He obtained his Power Engineering diploma, at Polytechnic University of Bucharest in 2006 and his Master of Science degree at the National Polytechnic Institute of Grenoble in 2007. He is currently $\mathrm{PhD}$ researcher in low voltage power systems at Grenoble Electrical Engineering Laboratory (G2ELab). His research interests are lighting systems and real time transient analysis in low voltage power systems.

Daniel Radu was born in 1975 in Bucharest, Romania. He obtained a Ph.D. degree in Electrical Engineering, in 2004, from the University "POLITEHNICA" of Bucharest, Romania. From 1998 to 2004 he has been assistant professor of Electrical Engineering at the same University. He is currently with Schneider Electric, France. His research interests include low voltage power systems transient analysis, power systems modelling and low voltage switching equipments design.

Seddik Bacha received his Engineer and Master from National Polytechnic Institute of Algiers respectively in 1982 and 1990. He joined the Laboratory of Electrical Engineering of Grenoble (LEG) and received his PhD and HDR respectively in 1993 and 1998. He is presently manager of Power System Group of LEG and Professor at the University Joseph Fourier of Grenoble. His main fields of interest are power electronics systems, modeling and control, power quality, renewable energy integration. 